\title{
Plasma levels of DDE/DDT and liver function in malaria control personnel 6 months after indoor residual spraying with DDT in northern Uganda, 2008
}

\author{
G S Bimenya, M Harabulema, J P Okot, Olwa Francis, Myers Lugemwa, A L Okwi
}

Objective. We investigated the relationship between plasma levels of dichlorodiphenyltrichloroethane (DDT) and liver function in malaria control personnel 6 months after one round of DDT indoor residual spraying (IRS).

Method. This was a cross-sectional study in the districts of Apac and Oyam of Lango, northern Uganda. Volunteers were clinically examined, and $5 \mathrm{ml}$ samples of venous blood were taken in heparinised tubes for a 6-month post-spray screening for DDT and plasma markers of liver function and internal organ disease. DDE/DDT was assayed using ELISA kits (Abraxis, USA); plasma enzyme activity concentrations of amylase, aspartate aminotransferase (AST), alanine aminotransferase (ALT) and gamma glutamyl transpeptidase (GGT) were analysed using routine clinical chemistryautomated methods (Konelab, Vantaa, Finland).
Results. All 96 plasma samples analysed for xenobiotics contained DDE/DDT in the empirical range of 24.00 - 128.00 parts per billion $(\mathrm{ppb})$ with a mean $(\mathrm{SD})$ of $77.00( \pm 26.00) \mathrm{ppb}$. All 119 plasma samples studied for the markers exhibited enzyme activity concentration values within the population reference ranges, with empirical means (SD) of amylase 71.86 (34.07), AST 23.83 (12.71), ALT 7.84 (10.01) and GGT 58.37 (62.68) $\mu \mathrm{g} / \mathrm{l}$.

Conclusion. Six months after IRS with DDT, the spray team had an average concentration of plasma DDE/DDT of 77 $\mathrm{ppb}$. This had no deleterious effect on liver function. We recommend continued use of DDT for IRS disease control in Uganda until better practical alternatives are available.

S Afr Med J 2010; 100: 118-121.

information collection and reporting on the use of DDT for disease vector control. ${ }^{2}$

Although animal models report dichlorodiphenyldichloroethylene (DDE)/dichlorodiphenyltrichloroethane (DDT) as a possible carcinogen, no such effects have been reported in humans. ${ }^{3}$ However, controversy exists concerning the liver: whereas some studies have reported impaired liver function, ${ }^{4}$ others reported an increase in the activity of liver enzymes, ${ }^{5}$ which could indicate liver disease such as hepatoma, or the usual enzyme catabolic induction typical of aromatic compounds, with no liver disease. ${ }^{6}$

Our objectives were to determine the concentration of DDE/ DDT in plasma samples from the spray team 6 months after their last spraying activity in northern Uganda in 2008, and to analyse plasma levels of biochemical markers of disease in blood from the spray team after exposure to DDT, so as to document acute/mid-term toxicity to internal organs, and especially liver function.

\section{Methods}

Approval for the study was granted by the Makerere University Faculty of Medicine Ethical and Research Committee. The project areas included Apac and Oyam districts and Lango sub-region (referred to as northern Uganda). Six months after the one IRS round of DDT in northern Uganda, the Ministry of Health's Malaria Control Programme clinician reviewed and physically examined the DDT spray team after obtaining informed and signed consent. Venous blood samples (of $5 \mathrm{ml}$ ) were collected into heparin 
Vacutainer tubes and delivered in cool boxes to the Department of Pathology, College of Health Sciences, Makerere University, for analysis for DDT and biochemical screening disease markers.

\section{Laboratory procedures}

The DDT in plasma was extracted with methanol and analysed using enzyme-linked immunosorbent assay (ELISA) kits.

The samples were processed according to the manufacturer's standard operating procedures and quality assurance instructions; during the reactions, colour that was inversely proportional to the concentration of DDE (the principal derivative of DDT) in the sample, developed; its intensity was translated and converted into concentration by comparison with that produced by the standards and controls supplied with the DDT reagent kits. The concentrations were read at 450 and $630 \mathrm{~nm}$ and printed automatically by the ELISA plate reader, the Stat FaxReg303 Plus.

Automated routine clinical chemistry methods were used in assaying for biochemical disease markers. Heparinised plasma samples were analysed for the concentration values of amylase, gamma glutamyl transpeptidase (GGT), and the transaminases (aspartate aminotransferase (AST) and alanine aminotransferase (ALT)) as biochemical screening markers for diseases of the internal organs, and specifically the liver.

\section{Results}

No abnormalities were detected on clinico-physical examination. No plasma abnormalities such as haemolysis, turbidity, chylosis, xanthochromia or jaundice were seen on visual inspection. The quantitative analytical results are presented at two levels: the primary level displays the main toxicological measure of interest (the DDT/DDE concentration in plasma samples from spray applicators 6 months after the one IRS round of DDT), and the secondary level displays the concentration values of the selected biochemical markers of disease.

\section{DDE/DDT concentration in post-spray plasma}

A total of 96 post-spray plasma samples were analysed for DDE/DDT concentration 6 months after the one round of DDT spraying (distribution shown in Fig. 1).

The DDE/DDT concentration in the post-spray plasma ranged empirically from 24 to 128, with a mean (SD) of 77 (26) $\mathrm{ppb}$; the spread was near normal but leptokurtic and positively skewed; as was expected, there was DDE/DDT in every postspray plasma sample analysed. The geometric mean was 71.81 ppb.

\section{Biochemical markers of diseases of the liver and internal} organs

A total of 119 plasma samples were analysed for the enzymes alpha-amylase, AST, ALT and GGT 6 months after spraying to

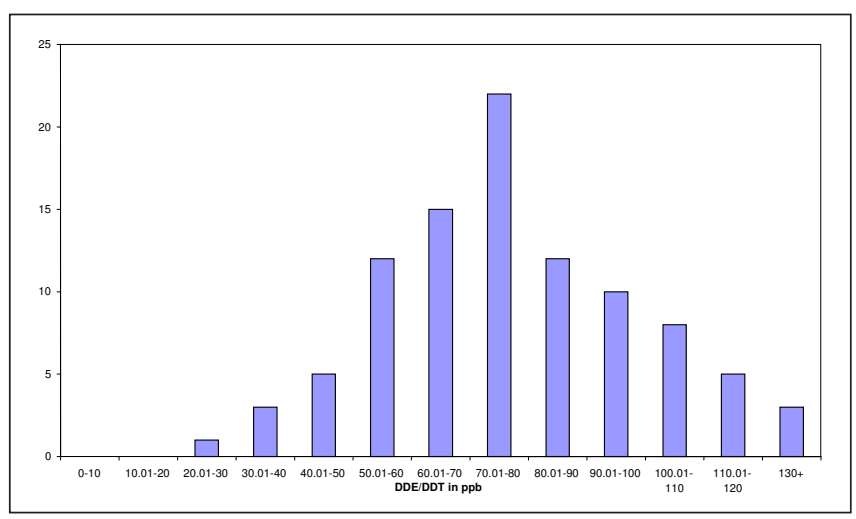

Fig. 1. DDDT concentration in post-spray plasma.

screen for acute/mid-term toxicity of DDE/DDT in the internal organs - the liver, pancreas, heart and biliary tract.

\section{Alpha-amylase concentration in post-spray plasma}

In 119 post-spray plasma samples analysed for alpha-amylase, the mean (SD) enzyme activity concentration was found to be $71.86(34.07)$ with a range of $16.80-199.50 \mu \mathrm{g} / \mathrm{l}$, as shown in Fig. 2.

The distribution was polymodal and within the reference range of up to $300 \mu \mathrm{g} / \mathrm{l}$. There was no alert value for any internal organ disease, especially not for acute pancreatitis as detected by plasma a-amylase in the post-spray plasma.

\section{AST concentration in post-spray plasma}

For post-spray AST activity concentration, 119 plasma samples were analysed; the mean (SD) was 25.83 (12.71); range 1.00 $63.90 \mu \mathrm{g} / \mathrm{l}$. The distribution was expectedly leptokurtic and positively skewed (Fig. 3).

The distribution of $88 \%$ of the results was within the reference range of up to $40 \mu \mathrm{g} / \mathrm{l} ; 12 \%$ were above the normal upper limit of $40 \mu \mathrm{g} / \mathrm{l}$ but within the inducible range of up to

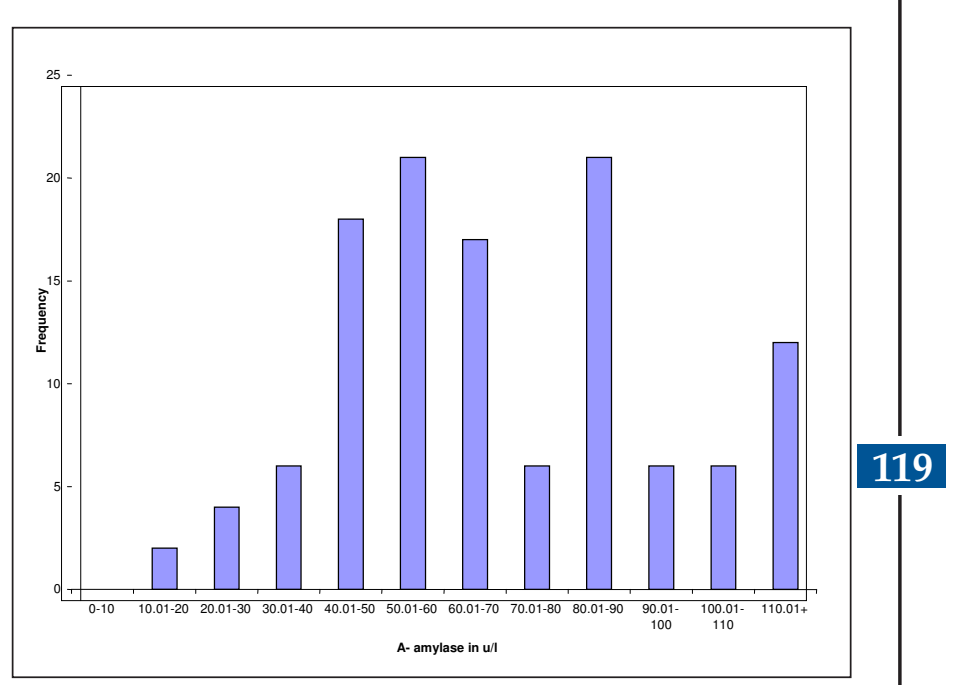

Fig. 2. Alpha-amylase enzyme activity concentration in post-spray plasma. 


\section{Original Articles}

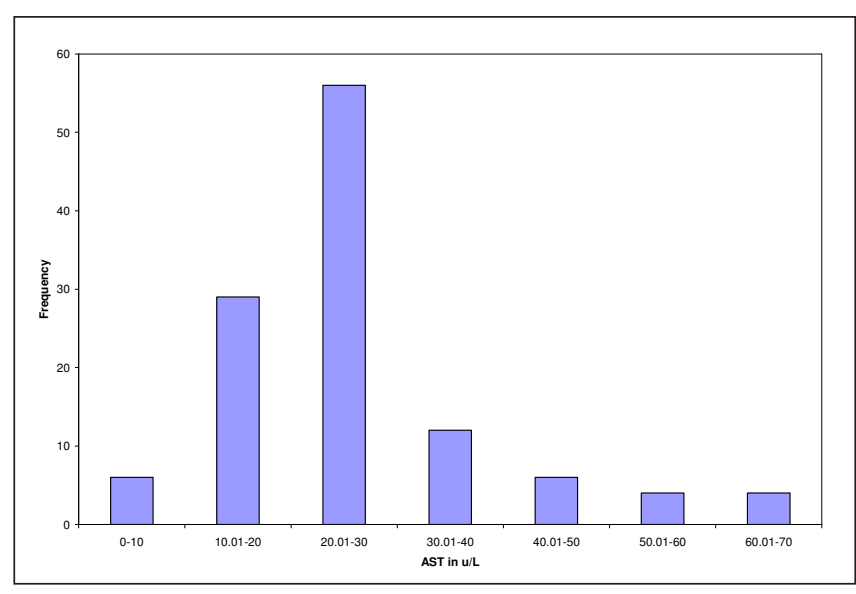

Fig. 3. AST enzyme activity concentration in post-spray plasma.

5 times the upper reference limit; there was no alert value for any internal organ disease, and specifically no hepatocellular or muscle damage, detected by high AST levels in the postspray samples.

\section{ALT enzyme activity concentration in post-spray plasma}

In screening for acute/mid-term DDT toxicity in internal organs such as the liver, 119 plasma samples were analysed for ALT, which resulted in a mean (SD) of 7.80 (5.01) and a range of $1.00-17.00 \mu \mathrm{g} / 1$. As expected, the spread was leptokurtic and positively skewed (Fig. 4).

The distribution was entirely within the reference range of up to $40 \mu \mathrm{g} / \mathrm{l}$, with no internal organ disease indicated or hepatocellular damage detected by high levels of ALT in the post-spray samples.

\section{GGT enzyme activity concentration in post-spray plasma}

For screening for internal organ disease, for liver disease in general and for biliary obstruction in particular, 119 plasma samples were analysed for GGT enzyme activity concentration. The mean (SD) values were 58.37 (62.68) and from the range $12.50-333.40 \mu \mathrm{g} / 1$. The spread was expectedly leptokurtic and positively skewed (Fig. 5).

Of the results, $75 \%$ were within the reference range of $<60$ $\mu \mathrm{g} / \mathrm{l} ; 30$ were within the drug-inducible range of below 5 times the upper limit of the reference range; and there was no liver disease or biliary tree abnormality overtly detected by high GGT levels in the post-spray plasma samples.

\section{Discussion}

Our study found that DDT successfully protected against mosquito bites, with no health complaints emerging from the clinical history evaluation. We documented a DDE/DDT plasma arithmetical mean of $77 \mathrm{ppb}$, with no biochemically alarming concomitant mean values (such as alpha amylase - 66.94, AST - 18.18, ALT - 9.33, and GGT - $31.05 \mu \mathrm{g} / \mathrm{l}$ ), indicating no disease associated with absorbed pesticide

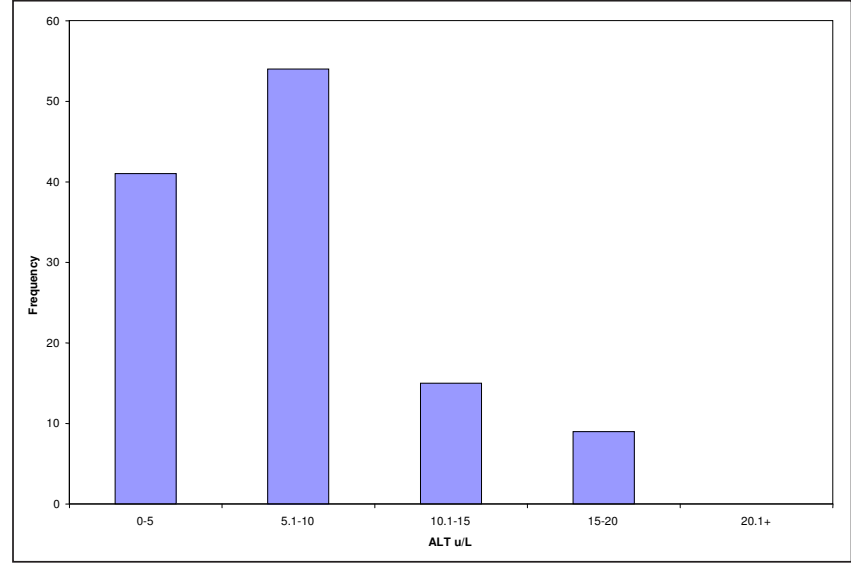

Fig. 4. ALT enzyme activity concentration in post-spray plasma.

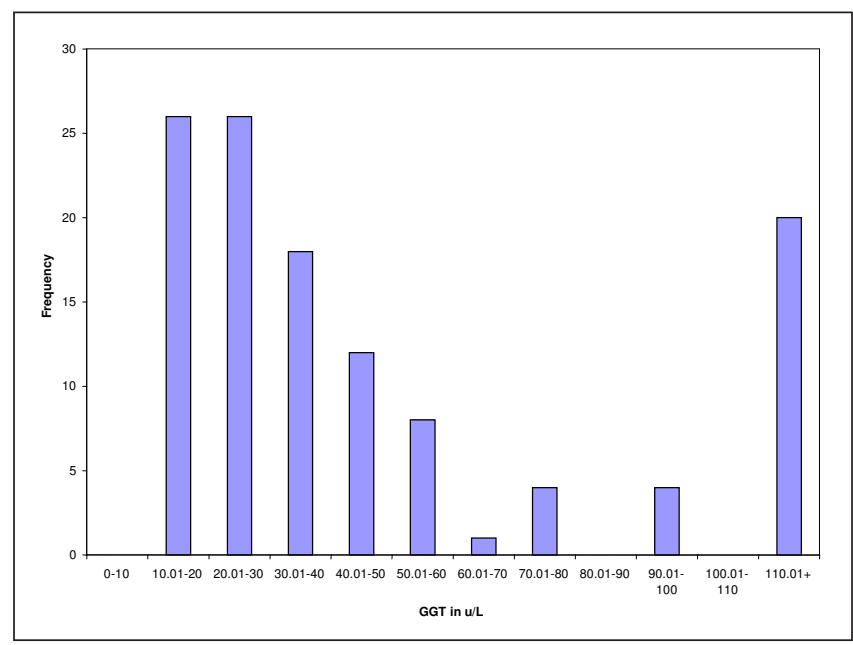

Fig. 5. GGT enzyme activity concentration in post-spray plasma.

subsequent to IRS operations. These results refute the association of DDT with acute or mid-term diseases of human internal organs, especially of the liver; they confirm the safety of DDT as used in IRS operations, and strongly commend the use of DDT for disease control according to WHO guidelines, especially where there are no better and readily available alternatives.

The ELISA technology used in this study was highly recommended for pesticide analyses, especially in developing countries, where it offers the advantages of cost-effectiveness, simplicity and speed over chromatographic methods. ${ }^{7}$

According to the Abraxis test kit manufacturers, the DDE/DDT assay kit used in this study in particular had cross-reactivity to organochlorine compounds at different percentages as follows: pp 'DDE - 100, pp`DDD - 46, op`DDD - 16, pp`DDT - 10, and op`DDE - 3.2. Whereas the method largely measured DDE (a metabolite of DDT), it did not appreciably measure DDT, and blood levels of DDE were, as usual, largely a measure of biotransformed DDT dependent inter alia on liver function and population genetics. We report and discuss them as DDE/DDT complex. 
Direct comparison of DDE/DDT versus organ function results with previous investigations is hampered by differences in study design, analytical facilities and age/population-related phenomena, especially among the young. ${ }^{8}$ Nevertheless, other studies had similar findings to ours. In 23 spray workers with 5.0 (6.3) years' continuous experience with DDT in Natal, ${ }^{9}$ where the sprayers were not required to wear protective aspirators, the mean DDE concentration was 129.0 (29.0) $\mu \mathrm{g} / \mathrm{l}$ (measured by more expensive and time-consuming gas chromatography with electron detector) - almost twice as high as our finding of $77 \mathrm{ppb}$. Similarly, in non-sprayers in KwaZulu-Natal, 29 adult residents of DDT-sprayed houses had an average accumulated plasma DDE concentration increase of $34.10 \mu \mathrm{g} / 1$ after 5 months' initial spraying. ${ }^{8}$ Again, this is close to our mean increase of $14.23 \mathrm{ppb}$ from the pre-spray background mean value of 62.92 obtained on recruitment ${ }^{10}$ of sprayers to the post-spray mean value of $77.15 \mathrm{ppb}$ obtained 6 months after the last DDT spraying activity. A study of occupational workers ${ }^{11}$ showed an increase of total DDT from a background mean value of $24 \mu \mathrm{g} / 1$ to a post-spray mean value of $130 \mu \mathrm{g} / 1$ over 5 months. ${ }^{11}$ Our mean concentration of DDE/DDT increased from the background mean (SD) of 63.00(19.00) to the post-spray value of $77.00(26.00) \mathrm{ppb}$ in the period March - October 2008. ${ }^{10}$ Compared with the results obtained in sprayers not protected with aspirators in Natal, ${ }^{9}$ we found little increase in DDE/DDT plasma concentration among the spray team. This finding is attributed inter alia to strict adherence to $\mathrm{WHO}$ recommendations for IRS ${ }^{12}$ of DDT. The comparatively little DDE/DDT detected in plasma in our study is a testimony that the spray team observed the documented safety precautions. ${ }^{13}$ This contradicts allegations of non-adherence to WHO spray guidelines, as lodged in court by DDT antagonists, ${ }^{14}$ and reflects the Uganda Malaria Control Programme preparedness in managing IRS.

In DDT applicators in South Africa, Bouwman et al. found that serum albumin, alkaline phosphatase, AST and GGT concentrations did not differ significantly from those of an age-matched control group, although the mean GGT value for the applicators was higher than the maximum of the laboratory normal range. ${ }^{9}$ Our ALT values fell entirely within the laboratory reference range of $<40 \mu \mathrm{g} / \mathrm{l}$. As ALT is a clinical indicator of hepatocellular damage, ostensibly normal values obtained in this work coupled with the good clinical history and uneventful clinical examination ruled out toxicological liver damage within the 6 months after DDT spraying.

Bouwman et al. ${ }^{9}$ again found values of ALT and GGT in the study group higher than in the control and the laboratory upper reference limit, respectively. Although expressing uncertainities, they still thought that the results suggested the possibility of risk to the health of the sprayers.
In our study, with the empirical range of AST being 0.70 $69.90 \mu \mathrm{g} / 1,12 \%$ of the plasma samples had values above the upper reference limit of $40 \mu \mathrm{g} / \mathrm{l}$. Similarly, with the empirical range of GGT being $12.50-333.40 \mu \mathrm{g} / \mathrm{l}, 25 \%$ of the samples had GGT above the population reference limit of $60 \mu \mathrm{g} / 1$ but within the aromatic drug/alcohol inducible range for the enzyme.

We caution that laboratory results should not be considered in isolation: clinical history and physical examination should ideally precede laboratory investigations. Our mild increases in enzyme activities may be explained as follows: besides enzyme induction caused by DDT as a xenobiotic, the AST mild increase was attributed to the muscular exercise involved in the spraying activity; that of amylase was the result of the starchrich millet-cassava diet made more prevalent by spray wages, and the GGT was because of increased consumption of lira-lira, a potent gin which is very popular in Northern Uganda.

This study in Northern Uganda in 2008, 6 months after only one round of DDT spraying, should for the first time provide the WHO with the essential information previously deemed missing, to evaluate exposure/response relationships to produce credible risk assessments of DDT use in IRS malaria control programmes.

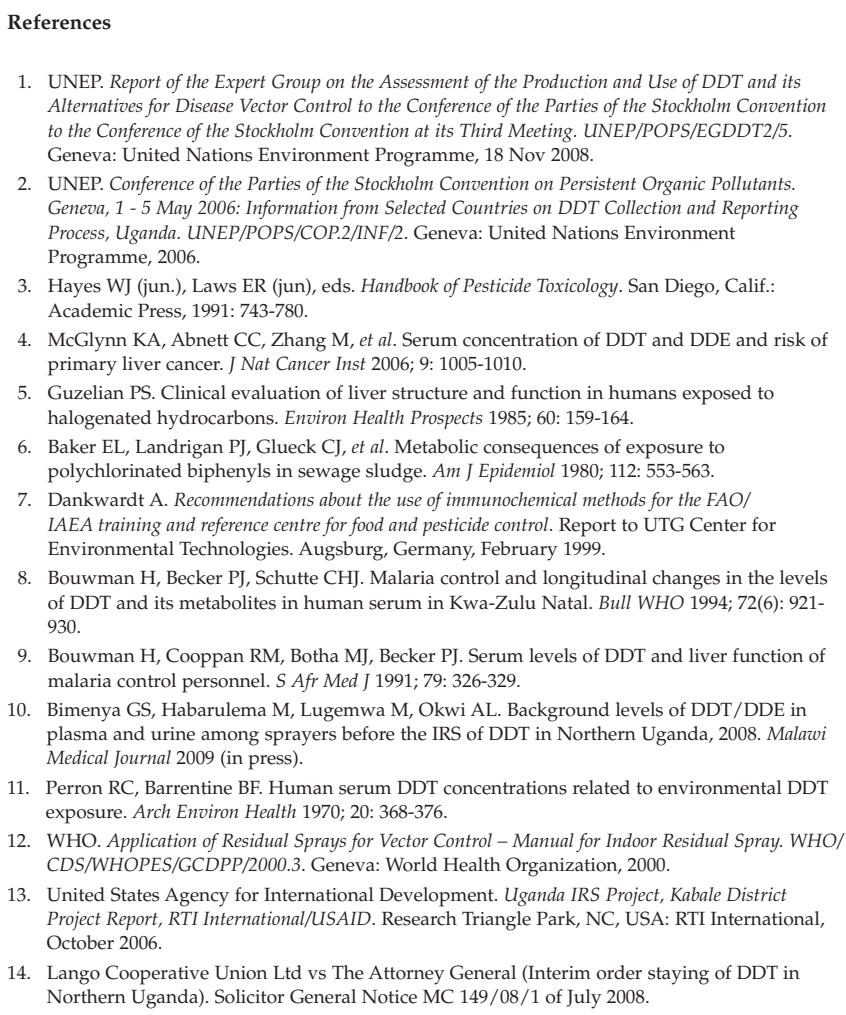

1. UNEP. Report of the Expert Group on the Assessment of the Production and Use of DDT and its Alternatives for Disease Vector Control to the Conference of the Parties of the Stockholm Conventio to the Conference of the Stockholm Convention at its Third Meeting. UNEP/POPS/EGDDT2/5. Geneva: United Nations Environment Programme, 18 Nov 2008.

2. UNEP. Conference of the Parties of the Stockholm Convention on Persistent Organic Pollutants. Geneva, 1 - 5 May 2006: Information from Selected Countries on DDT Collection and Reporting Process, Uganda. UNEP/POPS/COP.2/INF/2. Geneva: United Nations Environment Programme, 2006.

3. Hayes WJ (jun.), Laws ER (jun), eds. Handbook of Pesticide Toxicology. San Diego, Calif.: Academic Press, 1991: 743-780.

4. McGlynn KA, Abnett CC, Zhang M, et al. Serum concentration of DDT and DDE and risk of primary liver cancer. J Nat Cancer Inst 2006; 9: 1005-1010.

5. Guzelian PS. Clinical evaluation of liver structure and function in humans exposed to . Guzelian PS. Clinical evaluation of liver structure and function in hum
halogenated hydrocarbons. Environ Health Prospects 1985; 60: 159-164.

6. Baker EL, Landrigan PJ, Glueck CJ, et al. Metabolic consequences of exposure to polychlorinated biphenyls in sewage sludge. Am J Epidemiol 1980; 112: 553-563.

7. Dankwardt A. Recommendations about the use of immunochemical methods for the FAO/ IAEA training and reference centre for food and pesticide control. Report to UTG Center for Environmental Technologies. Augsburg, Germany, February 1999.

8. Bouwman $\mathrm{H}$, Becker PJ, Schutte CHJ. Malaria control and longitudinal changes in the level of DDT and its metabolites in human serum in Kwa-Zulu Natal. Bull WHO 1994; 72(6): 921 930.

9. Bouwman H, Cooppan RM, Botha MJ, Becker PJ. Serum levels of DDT and liver function of malaria control personnel. S Afr Med J 1991; 79: 326-329.

10. Bimenya GS, Habarulema M, Lugemwa M, Okwi AL. Background levels of DDT/DDE in plasma and urine among sprayers before the IRS of DDT in Northern Uganda, 2008. Malawi Medical Journal 2009 (in press).

11. Perron RC, Barrentine BF. Human serum DDT concentrations related to environmental DDT exposure. Arch Environ Health 1970; 20: 368-376.

12. WHO. Application of Residual Sprays for Vector Control - Manual for Indoor Residual Spray. WHO/ CDS/WHOPES/GCDPP/2000.3. Geneva: World Health Organization, 2000.

13. United States Agency for International Development. Uganda IRS Project, Kabale District Project Report, RTI International/USAID. Research Triangle Park, NC, USA: RTI International, October 2006.

14. Lango Cooperative Union Ltd vs The Attorney General (Interim order staying of DDT in Northern Uganda). Solicitor General Notice MC 149/08/1 of July 2008.

Accepted 24 August 2009. 